\title{
Leishmaniose tegumentar americana canina na zona rural do município de São Miguel, Rio Grande do Norte*
}

\section{Leishmaniasis Tegumentary American dog in rural area of the county of São Miguel, Rio Grande do Norte}

Francisco Rodrigues de Freitas Araújo, ${ }^{* *}$ Weibson Paz Pinheiro Andre, ${ }^{* *}$ Paulo Fernando Cisneiros da Costa Reis, ${ }^{* * * *}$ Taciana de Melo Fernandes, ${ }^{* * * *}$ Jael Soares Batista******

\begin{abstract}
Resumo
Leishmaniose Tegumentar Americana (LTA) constitui um problema de saúde pública emergente no Rio Grande do Norte, com relatos de casos humanos principalmente na Região Serrana do Alto Oeste do estado, uma vez que o cão tem um importante papel na manutenção da doença neste ambiente. O presente estudo teve por objetivo analisar prevalência da LTA canina em quatro localidades localizadas na Zona Rural do município de São Miguel, RN. Foram realizados exames clínicos e diagnóstico sorológico através das técnicas de ELISA e RIFI em 100 cães de diferentes sexo, idade e categorias companhia e de caça. Todas as localidades apresentaram cães soropositivos com resultados de $21 \%$ de positividade para a técnica de ELISA e $10 \%$ para a de RIFI. Maiores prevalências foram observadas em cães machos, assintomáticos, na faixa etária de dois anos de idade e de caça. O presente estudo confirma a importância epidemiológica como reservatório da infecção e poderá auxiliar nas medidas de prevenção e controle da doença no município.
\end{abstract}

Palavras-chave: cães, protozoário, sorodiagnóstico.

\begin{abstract}
American Cutaneous Leishmaniasis ( $A C L$ ) is an emerging public health problem in Rio Grande do Norte , with reports of human cases mainly in the mountainous region of the Upper Midwest state. Once the dog has an important role in maintaining the disease in this environment the present study aimed to evaluate the prevalence of canine ACL at four sites located in the Rural Area of São Miguel, RN . Clinical and serological diagnosis through ELISA and IFA in 100 dogs of different sex, age and company categories and hunting were performed. All towns had seropositive dogs with results of $21 \%$ positivity for ELISA and $10 \%$ for the IFA. Highest prevalence rates were observed in males, healthy dogs aged two years old and hunting. The present study confirms the epidemiological importance as a reservoir of infection and may assist in the prevention and control of disease in the city.
\end{abstract}

Keywords: dogs, protozoan, serodiagnosis.

\section{Introdução}

A Leishmaniose Tegumentar Americana (LTA) é uma doença infecciosa, endêmica, não contagiosa, causada por protozoários do gênero Leishmania, pertencente à família Trypanosomatidae, que acomete pele e mucosas. É primariamente uma enfermidade zoonótica, afetando animais domésticos, silvestres e o homem, predominando em regiões tropicais e subtropicais (Brasil, 2006; Luciano et al.; 2009).

O inseto vetor da LTA é um flebotomíneo do gênero Lutzomyia, conhecido popularmente como "mosquito-palha", "tatuíra", "birigui", "cangalha" e "cangalhinha" (Rey, 2001, Brasil, 2006).

A LTA é considerada pela Organização Mundial da Saúde (OMS) uma das seis mais importantes doenças infecciosas, sendo um problema de saúde pública em 88 países, distribuídos em quatro continentes (Américas, Europa, África e Ásia), com registro anual de 1 a 1,5 milhões de casos (Brasil, 2010).

A LTA, também conhecida como leishmaniose mucocutânea, úlcera de Bauru e ferida brava, distribui-se amplamente no continente americano, estendendo-se desde o sul dos Estados Unidos até o norte da Argentina (Lainson e Shaw; 1974).

Nas Américas, a LTA apresenta três formas clínicas principais: a leishmaniose cutânea, a leishmaniose mucocutânea e a leishmaniose difusa. No Brasil, a LTA permanece em expansão, e está cada vez mais associada às alterações ambientais (desmatamento e redução de matas primárias, especialmente as ciliares), à acelerada expansão das fronteiras agrícolas e ao desenvolvimento de outras atividades sinantrópicas como o ecoturismo (Araújo et al., 2008).

\footnotetext{
*Recebido em 12 de maio de 2014 e aceito em 23 de setembro de 2014.

**Médico-veterinário Autônomo, São Miguel - RN.

***Aluno de graduação em Medicina Veterinária, UFERSA, Mossoró, RN, Brasil.

****Médico Veterinário, UFERSA, Mossoró, RN, Brasil.

*****Doutoranda em Ciência Animal, UFERSA, Mossoró, RN, Brasil.

${ }^{\star * \star \star \star \star}$ Professor Doutor em Patologia Experimental do Departamento de Ciências Animais, UFERSA, Mossoró, RN, Brasil.
} 
A LTA vem sendo diagnosticada em todos os estados do país, inclusive na região Sul. As populações rurais do Norte, Nordeste e Centro-Oeste são as mais afetadas, sendo as regiões Norte e Nordeste responsáveis por cerca de $75 \%$ dos casos de LTA registrados no país (Marsden et al., 1985; Silveira et al., 2004)

A constatação da LTA sob as formas sintomática e subclínica em regiões onde também ocorrem casos humanos, sugere que o cão está presente na cadeia de transmissão (Santos et al., 2005), o que torna importante investigar a infecção por Leishmania sp. em cães, bem como relacioná-la com os casos humanos. Sendo assim, o objetivo deste trabalho foi analisar a prevalência da LTA canina na Zona Rural do município de São Miguel, Rio Grande do Norte.

\section{Material e métodos}

O trabalho foi realizado em quatro diferentes localidades na Zona Rural do Município de São Miguel (Olho d'Água Dantas, Barreiros, Agreste e São Pedro), localizado a $06^{\circ}-12^{\prime}-43^{\prime \prime}$ S $38^{\circ}-29^{\prime}-49^{\prime \prime}$, com altitude $679 \mathrm{~m}$ ao nível do mar e seu clima tropical. É um município brasileiro no interior do estado do Rio Grande do Norte, pertencente à Microrregião da Serra de São Miguel e Mesorregião do Oeste Potiguar. Localiza-se a oeste da capital do Estado, distância de $444 \mathrm{~km}$, sendo que $1,4214 \mathrm{~km}^{2}$ estão em perímetro urbano, e uma população de 22159 habitantes, sendo 14502 população urbana e 7657 em zona rural (IBGE, 2012), sendo então o $25^{\circ}$ município mais populoso do estado e primeiro de sua microrregião. A sede tem uma temperatura média anual de $27,1^{\circ} \mathrm{C}$ e a caatinga é a vegetação predominante no município.

Mediante autorização prévia dos proprietários, realizou-se o exame clínico de 100 cães, de diferentes sexos, idades e categorias - companhia e de caça, onde se avaliou o estado nutricional, dermatoses, lesões sugestivas de leishmaniose, tais como presença de úlceras nas orelhas, focinho ou bolsa escrotal, aumento de volume dos linfonodos, crescimento exagerado das unhas. Posteriormente, coletou-se entre 3 e $5 \mathrm{~mL}$ de sangue por punção venosa de cada animal, utilizando-se a veia braquiocefálica, acondicionando-o em tubos identificados individualmente sem anticoagulantes. Após retração do coágulo, o material foi centrifugado a 2000 rpm por 10 minutos para separação do soro e armazenado a $-20^{\circ} \mathrm{C}$, sendo posteriormente enviado ao laboratório de Bioquímica da Universidade Federal do Rio Grande do Norte.

Realizou-se pesquisa da presença de anticorpos antileishmaniose pelo método imunoenzimático (ELISA), utilizando-se extrato bruto de Leishmania chagasis isolado de um paciente humano que teve como princípio o conjugado humano lgG peroxidase na diluição de 1:2000. Foram consideradas positivas as amostras com densidade óptica média superior a 0,207 para a proteína rK-39.

A imunofluorescência indireta (RIFI) foi executada através de kit comercial para diagnóstico de Leishmania canina (BIOMAGUINHOS/FIOCRUZ) que utiliza como antígeno formas promastigotas de Leishmania. Na reação, considerou-se reagente titulação superior a 1:40, tomando como referência os soros controle positivo e negativo incluídos em cada lâmina.

\section{Resultados e discussão}

Das 100 amostras de sangue coletadas, 21 (21,0\%) foram reagentes para LTA por meio do teste de ELISA. Das amostras positivas no referido teste, 10 amostras foram positivas para RIFI (10,0\%). A prevalência variou em decorrência da localidade estudada, sendo o local Agreste com maior prevalência, com $52,38 \%$ para o teste de ELISA e $45,5 \%$ para RIFI, respectivamente (Tabelas 1 e 2 ).

Tabela 1: Soroprevalência da leishmaniose tegumentar americana em cães, por meio do teste de ELISA, por localidade do município de São Miguel, RN

\begin{tabular}{lccc}
\hline $\begin{array}{c}\text { Localidades do Município de } \\
\text { São Miguel }\end{array}$ & $\begin{array}{c}\mathbf{N}^{\circ} \text { de cães analisados } \\
\text { pelo método de ELISA }\end{array}$ & $\begin{array}{c}\mathbf{N}^{\circ} \text { de cães } \\
\text { soropositivos }\end{array}$ & Percentual (\%) \\
\hline Olho d,água Dantas & 39 & 5 & 23,81 \\
Barreiros & 10 & 2 & 9,52 \\
Agreste & 31 & 11 & 52,38 \\
São Pedro & 20 & 3 & 14,29 \\
Total de animais & 100 & 21 & 100 \\
\hline
\end{tabular}

Tabela 2: Soroprevalência da leishmaniose tegumentar americana em cães, por meio do teste de RIFI, por localidade do município de São Miguel, RN

\begin{tabular}{lccc}
\hline $\begin{array}{c}\text { Localidades do Município de } \\
\text { São Miguel }\end{array}$ & $\begin{array}{c}\mathbf{N}^{\circ} \text { de cães submetidos } \\
\text { a reteste RIFI }\end{array}$ & $\begin{array}{c}\mathbf{N}^{\circ} \text { de soropositivo } \\
\text { RIFI }\end{array}$ & $\begin{array}{c}\text { Percentual } \\
\mathbf{( \% )}\end{array}$ \\
\hline Olho d,água Dantas & 5 & 3 & 14,2 \\
Barreiros & 2 & 1 & 4,76 \\
Agreste & 11 & 5 & 23,8 \\
São Pedro & 3 & 1 & 4,76 \\
Total de animais & 21 & 10 & 47,6 \\
\hline
\end{tabular}

Leishmaniose Tegumentar Americana (LTA) em humanos é um problema de saúde emergente no Rio Grande do Norte, principalmente na Região Serrana do Alto Oeste do estado, fronteira com as regiões endêmicas dos estados do Ceará e da Paraíba. Nesta região a ocorrência de LTA apresenta flutuação ao longo dos anos, porém nos anos de 2009 e 2010 os casos cresceram de forma considerável, principalmente nos municípios de São Miguel e Venha Ver (Lima, 2010). A realização de inquéritos sorológicos caninos, além de sua função de controle do reservatório canino em extensas áreas, tem papel fundamental na detecção de focos silenciosos da doença e na delimitação de regiões ou setores de maior prevalência, onde a execução das medidas de controle se faz necessária (Julião 
et al., 2007). A ocorrência de cães sorologicamente positivos para LTA, tal como verificado no presente estudo, confirma o papel do cão no ciclo de transmissão do parasita e sugere que a área estudada seja endêmica e com situação epidemiológica de alta transmissibilidade. Isso alerta quanto a necessidades de diagnósticos periódicos da LTA, bem como da realização de medidas de controle da doença, como por exemplo, uma tentativa de interrupção do ciclo de transmissão do parasita, uma vez que os caninos são fontes de infecção a doença em humanos.

Dentre as amostras analisadas houve predominância sexual e etária, sendo as maiores prevalências em cães machos na faixa etária de dois anos de idade (Tabelas 3 e 4). Analisando os dados quanto à categoria dos animais foi possível observar que $71,42 \%$ e $80 \%$, respectivamente para os testes de ELISA e RIFI, eram cães de caça (Tabela 5).

Tabela 3: Soroprevalência de leishmaniose tegumentar americana em cães do município de São Miguel, diagnosticado pelo teste de ELISA e RIFI, segundo sexo

\begin{tabular}{lcccc}
\hline Sexo & $\begin{array}{c}\mathbf{N}^{\circ} \text { de } \\
\text { animais }\end{array}$ & $\begin{array}{c}\text { Percentagem } \\
\text { ELISA }\end{array}$ & $\begin{array}{c}\mathbf{N}^{\circ} \text { de } \\
\text { animais }\end{array}$ & $\begin{array}{c}\text { Percentual } \\
\text { RIFI }\end{array}$ \\
Macho & 17 & $80.95 \%$ & 9 & $90 \%$ \\
Fêmea & 4 & $19.05 \%$ & 1 & $10 \%$ \\
Total & 21 & 100 & 10 & 100 \\
\hline
\end{tabular}

Tabela 4: Soroprevalência da leishmaniose tegumentar americana em cães do município de São Miguel, diagnosticado pelo teste de ELISA e RIFI, segundo a idade

\begin{tabular}{ccccc}
\hline & $\begin{array}{c}\mathbf{N}^{\mathbf{0}} \\
\text { animais }\end{array}$ & $\begin{array}{c}\text { (\%) positivos } \\
\text { ELISA }\end{array}$ & $\begin{array}{c}\mathbf{N}^{\mathbf{2}} \\
\text { animais }\end{array}$ & $\begin{array}{c}\text { (\%) positivos } \\
\text { RIFI }\end{array}$ \\
\hline 1 & 3 & 14.28 & 2 & 20 \\
2 & 10 & 47.61 & 5 & 50 \\
3 & 3 & 14.28 & 2 & 20 \\
4 & 2 & 9.52 & 1 & 10 \\
5 & 1 & 4.77 & - & - \\
6 & 1 & 4.77 & - & - \\
Acima de 6 & 1 & 4.77 & - & - \\
Total & 21 & 100 & 10 & 100 \\
\hline
\end{tabular}

A maior prevalência da LTA em cães machos está de acordo com Naveda et al. (2006), que afirmam que os machos são mais frequentemente parasitados. A maior prevalência da LTA em cães adultos também está de acordo com Arias et al. (1996) que demonstram uma predisposição dos cães adultos em adquirirem a doença, associada provavelmente ao longo período de incubação. De acordo com os dados obtidos, observa-se que as variáveis, sexo e idade são considerados fatores de risco da leishmaniose canina no município de São Miguel.
Tabela 5: Soroprevalência da leishmaniose tegumentar americana em cães do município de São Miguel, diagnosticado pelo teste de ELISA e RIFI, segundo as categorias de cães de caça e domiciliar

\begin{tabular}{lcccc}
\hline Categorias & $\begin{array}{c}\mathbf{N}^{\circ} \\
\text { animais }\end{array}$ & $\begin{array}{c}\text { Percentual } \\
\text { ELISA }\end{array}$ & $\begin{array}{c}\mathbf{N}^{\circ} \\
\text { animais }\end{array}$ & $\begin{array}{c}\text { Percentual } \\
\text { RIFI }\end{array}$ \\
\hline Caça & 15 & 71.42 & 8 & 80 \\
Domiciliar & 6 & 28.58 & 2 & 20 \\
Total & 21 & 100 & 10 & 100 \\
\hline
\end{tabular}

A observação de maior prevalência de LTA em cães de caça também condiz com Uchôa et al. (2001), sendo tal fato provavelmente associado à maior exposição do cão de caça e consequente maior contato desses animais com o vetor e animais silvestres que são reservatórios para a enfermidades, havendo, portanto, maior risco a infecção do que os cães de companhia, criados em ambiente domiciliar. A maioria dos cães analisados era sem raça definida e não apresentavam sinais clínicos compatíveis com a doença ou foram oligossintomáticos, apresentando apenas alopecia. A ocorrência de cães com sorologia positiva para LTA, porém sem lesões, assume grande importância epidemiológica, uma vez que segundo Cardoso et al. (1989), a inexistência de lesões suspeitas em cães de áreas endêmicas de LTA humana não significa que a doença canina não esteja presente. A ocorrência de animais sorologicamente positivos sem lesões reafirma a importância dos inquéritos sorológicos caninos para identificação de animais positivos assintomáticos.

No presente estudo foram realizados dois testes sorológicos (ELISA e RIFI), para identificação de animais positivos, conforme recomendação do Ministério da Saúde que preconiza que para avaliação da soroprevalência, seja realizado o ensaio imunoenzimático (ELISA), por apresentarem alta sensibilidade e especificidade, baixo custo e fácil execução associado ao teste de RIFI (Brasil, 2006). Alguns autores consideram a RIFI como teste padrão, com emprego universal, onde epidemiologicamente títulos iguais ou superiores a 1/40 devem ser considerados positivos. No entanto, a utilização de duas técnicas na busca de um único diagnóstico em um mesmo inquérito é útil para uma triagem de casos ou em inquéritos epidemiológicos, permitindo a execução de uma sorologia confiável (Costa e Vieira, 2001).

\section{Conclusão}

AZona Rural do município de São Miguel possui alta prevalência de cães sorologicamente positivos para Leishmaniose Tegumentar Americana, sendo a maior prevalência observada em cães adultos $(85,72 \%)$, machos $(80,95 \%)$ e de caça $(71,42 \%)$. Dessa forma, o cão provavelmente funciona como reservatório da infecção para população humana. 


\section{Referências}

ARAÚJO, N.S.; MACHADO, M.I.; ROCHA, A.; CURY, M.C. Aspectos clínicos e histopatológicos da Leishmaniose Tegumentar Americana em pacientes atendidos no hospital de clínicas da Universidade Federal de Uberlândia, MG, Brasil. Revista de Patologia Tropical. n. 1, v. 37, p. 23-31, 2008.

ARIAS, J.R.; MONTEIRO, P.; ZICKER, F. The re-emergence of visceral leishmaniasis in Brasil. Emerging Infectious Diseases, $\mathrm{n}$. 2, p. 145-146, 1996.

BRASIL. MINISTÉRIO DA SAÚDE. Secretaria de Vigilância em Saúde. Departamento de Vigilância Epidemiológica. Manual de vigilância e controle da leishmaniose visceral. Série A. Brasília. Normas e Manuais Técnicos. Brasília, DF: 2006. Disponível em: http://bvsms.saude.gov.br/bvs/publicacoes/manual_vigilancia_ controle_leishmaniose_visceral.pdf

BRASIL. MINISTÉRIO DA SAÚDE. Secretaria de Vigilância em Saúde. Departamento de Vigilância Epidemiológica. Manual de Vigilância da Leishmaniose Tegumentar Americana. Série A. Normas e Manuais Técnicos. Brasília, DF: 2010. Disponível em:http: //bvsms.saude.gov.br/bvs/publicacoes/ manual_ vigilancia_leishmaniose_tegumentar_americana.pdf

CARDOSO, S.R.A.; MACHADO, M.I.; COSTA-CRUZ, J.M.; GONCALVES, M.R. F.; STUTZ, W.H. Leishmaniose tegumentar canina no Município de Uberlândia, Minas Gerais: Diagnostico clinico e sorológico de cães naturalmente infectados. Revista Centro Ciência Biomédica Universidade Federal Uberlândia, n. 5, p. 14-21, 1989.

COSTA, C.H.N.; VIEIRA, J.B.F. Mudanças no controle da leishmaniose visceral no Brasil. Revista da Sociedade Brasileira de Medicina Tropical, v. 34, n. 2, p. 223-228, 2001.

IBGE. Relatório de Estimativas da população residente nos municípios brasileiros. Instituto Brasileiro de Geografia e Estatística (2012).

JULIÃO, F.S.; SOUZA, B.M.P.S.; FREITAS, D.S.; OLIVEIRA, L.S.; LARANJEIRA, D.F.; DIAS-LIMA, A.G.; SOUZA, V.M.M.; BARROUIN-MELO, S.M.; MOREIRA JR, E.D.; PAULE, B.J.A.; FRANKE, C.R. Investigação de áreas de risco como metodologia complementar ao controle da leishmaniose visceral canina. Pesquisa Veterinária Brasileira, n. 27, p. 319-324, 2007.

LAINSON, R.; SHAW, J.J. Leishmaniasis y las leishmanias del nuevo mundo, con particular referencia en Brasil. Bol Oficina Sanit Panam, v. 76, n. 93, 1974.
LIMA, I.D. Relatório da situação da leishmaniose tegumentar americana na região serrana do RN, em janeiro de 2010. Relatório Secretaria de Estado de Saúde Pública. Natal, RN, 2010. Disponível em: http://WWW.dei.rn.gov.br/.../saúde.../ relatório_situacao_rn_2010.pdf

LUCIANO, R.M.; LUCHEIS, S.B.; TRONCARELLI, M.Z.; LUCIANO, D.M.; LANGONI, H. Avaliação da reatividade cruzada entre antígenos de Leishmania spp e Trypanosoma cruzi na resposta sorológica de cães pela técnica de imunofluorescência indireta (RIFI). Braz. J. Vet. Res. Anim. Sci., v. 46, n. 3, 2009.

NAVEDA, L.A.B.; MOREIRA, E.C.; MACHADO, J.G.; MORAES, J.R.C.; MARCELINO, A.P. Aspectos epidemiológicos da leishmaniose visceral canina no município de Pedro Leopoldo, Minas Gerais. Arquivo Brasileiro de Medicina Veterinária e Zootecnia, n. 58, p. 988-993, 2006.

MARSDEN, P.D; JONES, T.C. Clinical manifestations, diagnosis and treatment of leishmaniasis. Leishmaniasis. Human Parasitic Diseases, v.1, p. 183-198, 1985.

REY, L. Leishamnia e leishmaniasis: os parasitos, p. 215-226. In: REY, L. (Ed.). Parasitologia. 3. ed. Rio de Janeiro: Guanabara Koogan, 2001.

SANTOS, G.P.L.; SANAVRIA, A.; MARZOCHI, M.C.A. et al. Prevalência da infecção canina em áreas endêmicas de leishmaniose tegumentar americana, no município de Paracambi, Estado do Rio de Janeiro, no período entre 1992 e 1993. Revista Sociedade Brasileira de Medicina Tropical, v. 38, p.161-166, 2005.

SILVEIRA, F.T.; LAINSON, R.; CORBETT, C.E. Clinical and Immunopathological Spectrum of American Cutaneous Leishmaniasis with Special Reference to the Disease in Amazonian Brazil - A Review. Memórias do Instituto Oswaldo Cruz, v. 99, n. 3, p. 239-251, 2004.

UCHÔA, C.M.A.; SERRA, C.M.B.; DUARTE, R.; MAGALHÃES, C.M.; SILVA, R.M.; THEOPHILO, F.; FIGLIUOLO, L.P.; HORTA, F.T.; MADEIRA, M.F. Aspectos sorológicos e epidemiológicos da leishmaniose tegumentar americana em Maricá, Rio de Janeiro, Brasil. Revista da Sociedade Brasileira de Medicina Tropical, n. 34, v. 6, p. 563-568, 2001. 